\title{
P38 MAPK Inhibits Autophagy and Promotes Hepatic Stellate Cells Inflammatory Responses via Atg13 During Acute-On-Chronic Liver Failure
}

Zhen Tian ( $\square$ tianzhen@xjtufh.edu.cn )

The First Affiliated Hospital of Xi'an Jiaotong University https://orcid.org/0000-0001-7475-9472

Naijuan Yao

The First Affiliated Hospital of Xi'an Jiaotong University

\section{Yuchao Wu}

The First Affiliated Hospital of Xi'an Jiaotong University

Fei Wang

The First Affiliated Hospital of Xi'an Jiaotong University

\section{Yingren Zhao}

The First Affiliated Hospital of Xi'an Jiaotong University

\section{Research Article}

Keywords: Atg13, p38 MAPK, autophagy, hepatic stellate cells, acute-on-chronic liver failure

Posted Date: April 30th, 2021

DOI: https://doi.org/10.21203/rs.3.rs-436479/v1

License: (c) (1) This work is licensed under a Creative Commons Attribution 4.0 International License. Read Full License 


\section{Abstract}

Inflammation plays a critical role in disease pathogenesis of acute-on-chronic liver failure (ACLF). Atg13 is a vital regulatory component of ULK1 complex, which plays an essential role in initiation of autophagy, and autophagy has been connected to hepatic inflammation. The aim of this study is to evaluate how autophagy regulates hepatic stellate cells (HSCs) inflammatory responses via Atg13 during ACLF. Clinical data were collected from ACLF patients, and surgical resected paraffin embedded human ACLF liver tissues specimens were collected. Inflammation and autophagy were investigated by immunoblot analysis in HSCs treated with lipopolysaccharide (LPS). Co-immunoprecipitation were used to investigate interaction of Atg13 and ULK1. Our data exhibit that serum LPS is positively associated with disease severity in ACLF patients, and p38 MAPK is overexpressed in ACLF liver tissues. Inflammatory factors are up-regulated via the activation of p38 MAPK and the inhibition of the autophagy in LX-2 cells. Furthermore, we illuminate in the vitro study that LPS triggers p38 MAPK activity, resulting in phosphorylation of Atg13, inhibition of Atg13-ULK1 interaction and autophagy. This study highlights a molecular mechanism that LPS promotes inflammation through inhibition of autophagy in HSCs via Atg13, and provides a new understanding into the mechanistic of inflammatory process of severe hepatitis and a novel strategy for ACLF treatment.

\section{Introduction}

Liver failure, including acute, chronic, and acute-on-chronic liver failure, is a rare but dramatic clinical syndrome characterized by massive hepatocyte death and overactivation of hepatic inflammation[1]. Acute-on-chronic liver failure (ACLF), characterized by an acute deterioration of liver function in patients with pre-existing chronic liver disease, usually results in hepatocellular dysfunction and carries a high mortality rate[2]. Apart from liver transplantation, few effective therapies are available, and ACLF continues to be a huge therapeutic challenge[3]. The precise molecular mechanisms for the pathogenesis of ACLF have not been clarified, exploring ACLF-associated molecules may enable the development of strategies to improve the prognosis for patients with ACLF.

Hepatic stellate cells (HSCs) are resident mesenchymal cells that have features of fibroblasts and pericytes, and account for $15 \%$ of total resident cells in normal human liver[4]. HSCs are key nonparenchymal components in the sinusoid with multiple functions[5]. Some recent studies have revealed that activated HSCs may release inflammatory cytokines like interleukin (IL)-1 $\beta$ and IL-18, and HSCs inflammation has been shown participated in the pathogenesis of several liver diseases: HSCs of murine or human origin produce inflammatory cytokines promoting hepatocellular carcinoma and immune-mediated hepatitis[6], our previous study has shown that HSCs inflammation participates in disease pathogenesis of ALF[7].

Autophagy is a conserved process by which cytoplasmic components, including damaged proteins and organelles, are degraded by lysosomes[8]. Autophagy and inflammation are highly intertwined cellular process. Autophagy suppresses proinflammatory process and inflammasome activity[9]. Decreased 
autophagy was found leading to elevated hepatic inflammation promoting the progression of alcoholic liver disease[10], and LPS \& D-GalN induced liver injury in mice[11]. Inflammatory cytokines also function to reciprocally control autophagy[9]. However, the mechanisms by which inflammatory signals specifically relieve the negative suppression by autophagy on HSCs inflammation during ACLF remains unknown.

Mitogen-activated protein kinase (MAPK), including p38, ERK and c-JNK are members of a ubiquitous protein serine/threonine kinase family responsible for signal transduction in eukaryotic organisms[12]. MAPK activation is implicated in the production of many inflammatory mediators[13]. Mammalian genomes encode four distinct p38 MAPK isoforms, including $a, \beta, \gamma$ and $\delta$. MAPK p38 $\alpha$ and $\beta$ are commonly activated by stressful or pro-inflammatory stimuli[14]. Most studies of p38 MAPK have focused on its functions in inflammatory cells during the pathogenesis of inflammation dependent diseases, including rheumatoid arthritis, Crohn's disease, psoriasis and asthma[15, 16].

In the present study, we show that p38 MAPK plays an essential role in relieving autophagic control in response to inflammatory signal. We found elevated expression of p38 MAPK in liver tissues from patients with ACLF. Stimulation of LPS inhibits autophagy via p38 MAPK, this inhibition is necessary for LPS-induced inflammasome activation in LX2 cells. We show that p38 MAPK directly interacts with Atg13, phosphorylates Atg13 and reduces Atg13-ULK1 interaction.

\section{Materials And Methods}

\subsection{Patients}

From January 2016 to September 2017, a total of 56 patients diagnosed with ACLF were enrolled in our study at the First Affiliated Hospital of Xi'an Jiaotong University, Shaanxi, China. All participants provided written informed consent, depending on the patient's altered mental status, and the study was approved by the Research Ethics Committee of the First Affiliated Hospital of Xi'an Jiaotong University. Patients were diagnosed with ACLF based on the criteria of Asian Pacific Association for the Study of the Liver (APASL): 1) serum bilirubin $\geq 85 \mathrm{~mol} / \mathrm{L} ; 2$ ) INR $\geq 1.5$ or prothrombin activity $\leq 40 \%$; 3 ) any degree of encephalopathy and/or clinical ascites within 4 weeks; 4 ) and an evidence of ongoing chronic liver diseases. Patients who were diagnosed with ACLF and aged 18 to 75 years were included. We calculated the Model for End-Stage Liver Disease (MELD) score using the standard formula: $11.2 * \ln ($ INR $)+9.57 * \ln$ (creatinine, in $\mathrm{mg}$ per decilitre) $+3.78 *$ In (bilirubin, in mg per decilitre), with a lower limit of 1 for all variables. During the same period, age- and sex-matched healthy and cirrhotic participants were recruited as controls.

\subsection{Antibodies and reagents}

LPS, SB230580, bafilomycin-A1 (Baf A1), rapamycin was purchased from Sigma-Aldrich. The following primary antibodies were used: anti-P38a MAPK (\#9217, Cell Signaling), anti-phospho-P38 MAPK (\#9216, Cell Signaling), anti-NLRP3 (\#15101, Cell Signaling), anti-IL1 (\#12703, Cell Signaling), anti-Beclin1 
(\#4122, Cell Signaling), anti-LC3 (\#8899, Cell Signaling), anti-P62 (\#5114, Cell Signaling), anti-Myc (\#2276, Cell Signaling), anti-Flag (\#14793, Cell Signaling) and $\beta$-Actin as a loading control (\#4970, Cell Signaling).

\subsection{Estimation of LPS and IL-1 $\beta$}

Serum LPS levels were measured by using a Limulus Amebocyte Lysate (LAL) commercial test kit (Xiamen Houshiji, Ltd, Xiamen, China), and Secretion IL-1 $\beta$ levels were detected by utilizing Human IL-1 beta Quantikine ELISA Kit (SLB50, R\&D Systems) according to the manufacture's protocol. Samples and standards were run in duplicate.

\subsection{Histological sampling}

We collected surgical resected paraffin-embedded human ACLF liver tissues specimens ( 5 cases) and cirrhotic liver tissue specimens ( 5 cases) from the Department of Pathology, the First Affiliated Hospital of Xi'an Jiaotong University, with the approval of the Institutional Review Board. Immunoreactions were performed on selected liver sections. Antigens were detected by one of the following primary antibodies, followed by appropriate secondary antibodies: anti-p38 (\#8690, Cell Signaling Technology, Danvers, MA, USA). The slides were then observed under a Nikon Eclipse microscope (Tokyo, Japan) coupled to a digital camera.

\subsection{Cells culture}

The human hepatic stellate cells LX2 cells, HEK-293 cells were cultured in Dulbecco's modified Eagle's medium and THP- 1 cells were cultured in RPMI- 1640 medium supplemented with $10 \%$ fetal bovine plasma and $2 \mathrm{mM} \mathrm{L-glutamine} \mathrm{at} 37^{\circ} \mathrm{C}$ in a $95 \%$ air, $5 \% \mathrm{CO}_{2}$-humidified atmosphere. Cells were trypsinized, and $5 \times 10^{5}$ cells were seeded onto plastic dishes and then treated with LPS, SB203580, Rapamycin or Bafilomycin A1.

\subsection{Plasmid transfection.}

HEK-293 cells were transfected with plasmids by using Lipofectamine 2000 reagent (Invitrogen). Wholecell lysates were prepared after $48 \mathrm{~h}$ plasmid transfection.

\subsection{Co-immunoprecipitation assay for protein binding}

Cells lysates were prepared in lysis buffer, and incubated with ProteinA/G-Sepharose beads at $4{ }^{\circ} \mathrm{C}$ for 3 hours. Pre-cleared lysates were incubated with appropriate antibody at $4{ }^{\circ} \mathrm{C}$ for 12 hours with gentle rotation. Protein-A/G-Sepharose beads were added and incubated for 3 hours. Immunoprecipitants were collected by centrifugation, washed five times with lysis buffer, heated at $100{ }^{\circ} \mathrm{C}$ for 5 min and subjected to SDS-PAGE.

\subsection{Immunoblotting}

Protein extracts were prepared from cells by RIPA Lysis Buffer supplemented with Complete EDTA-free protease inhibitor cocktail tablets (Roche Applied Science, Basel, Switzerland) and phosphatase inhibitor 
cocktails (Sigma-Aldrich). Protein samples $(50 \mu \mathrm{g})$ were loaded onto SDS-PAGE gels and transferred onto PVDF membranes. After blocking in 5\% evaporated milk at room temperature for $2 \mathrm{~h}$, the membranes were then incubated with the indicated primary antibodies in $5 \%$ evaporated milk in TBS plus $0.1 \%$ Tween 20 overnight at $4^{\circ} \mathrm{C}$. Signals were developed using a chemiluminescent substrate and visualized through X-ray films.

\subsection{Statistical analysis}

The results are expressed as the means \pm standard deviation. Statistical analysis was performed using SPSS software 13.0 (SPSS, Inc., Chicago, IL, USA). The Shapiro-Wilk test and Levene statistic were used to evaluate the normality and homogeneity of the variance, respectively. According to the situation, t-tests or Mann-Whitney U tests were used to evaluate differences between two groups; correlations between two quantitative groups were analyzed with Pearson or Spearman correlation tests. The $\chi 2$ test was used for comparisons between two groups. The reported P-values are two-sided, and P-values $<0.05$ were considered statistically significant.

\section{Results}

\subsection{Serum LPS level increased in ACLF}

Liver failure is an inflammation-mediated hepatocellular injury process, LPS levels in serum is elevated in patients with ALF and ACLF due to increased gut permeability. Here we investigated serum LPS levels in patients with ACLF (Table 1). We found a significant increase in serum LPS concentration in patients with ACLF compared to cirrhotic patients $(177.5 \pm 64.52$ vs $5.830 \pm 0.3019, P<0.05)$ (Fig. 1A). Previously, we found that MELD score $>25$ was associated with short-term mortality in ALF patients. We then divided these ACLF patients into a low-risk group (MELD score $\leq 25)$ or high-risk group (MELD score > 25).

Patients in the high-risk group presented with higher LPS levels compared to patients in the low-risk group $(217.0 \pm 70.56$ vs $83.68 \pm 31.79, P<0.05)$ (Fig. 1B). Furthermore, a positive correlation was observed when the LPS level was correlated with the MELD score $\left(R^{2}=0.5164, P<0.01\right)$ (Fig. 1C). 
Table 1

Demographic data and clinical characteristics of controls and ACLF patients

\begin{tabular}{|c|c|c|c|}
\hline Parameter & Healthy Control & Cirrhosis & ACLF \\
\hline & $(n=30)$ & $(n=30)$ & $(n=56)$ \\
\hline Age (yr) & $30.40 \pm 6.96$ & $37.18 \pm 4.49$ & $38.58 \pm 5.19$ \\
\hline Gender (M/F) & $26 / 4$ & $26 / 4$ & $50 / 6$ \\
\hline PTA (\%) & $85.29 \pm 12.58$ & $73.26 \pm 17.53$ & $32.26 \pm 11.97$ \\
\hline $\mathrm{FIB}(\mathrm{g} / \mathrm{L})$ & $3.04 \pm 0.52$ & $2.95 \pm 0.66$ & $2.65 \pm 0.93$ \\
\hline INR & $1.07 \pm 0.07$ & $1.17 \pm 0.15$ & $2.12 \pm 0.98$ \\
\hline WBC $(1 \times 109 / L)$ & $5.78 \pm 1.31$ & $4.21 \pm 1.63$ & $7.08 \pm 3.50$ \\
\hline PLT (1×109/L) & $229.40 \pm 37.80$ & $116.43 \pm 54.32$ & $95.82 \pm 52.25$ \\
\hline ALT (U/L) & $22.33 \pm 14.43$ & $34.81 \pm 20.36$ & $620.38 \pm 835.14$ \\
\hline GLU (mM) & $4.07 \pm 0.29$ & $4.38 \pm 0.43$ & $6.57 \pm 3.13$ \\
\hline TBIL $(\mu \mathrm{M})$ & $8.17 \pm 2.47$ & $18.32 \pm 8.67$ & $304.09 \pm 137.94$ \\
\hline CHOL (mM) & $3.58 \pm 0.73$ & $3.32 \pm 066$ & $2.25 \pm 0.75$ \\
\hline CREA $(\mu \mathrm{M})$ & $50.47 \pm 10.73$ & $54.32 \pm 12.67$ & $78.91 \pm 15.31$ \\
\hline $\mathrm{Na}(\mathrm{mM})$ & $140.80 \pm 2.24$ & $141.62 \pm 2.54$ & $135.32 \pm 14.87$ \\
\hline MELD & & & $23.82 \pm 6.71$ \\
\hline \multicolumn{4}{|c|}{$\begin{array}{l}\text { PTA, prothrombin activity; FIB, fibrinogen; INR, international normalized ratio; WBC, white blood cell } \\
\text { count; PLT, platelet count; ALT, alanine aminotransferase; GLU, glucose; TBIL, total bilirubin; CHOL, } \\
\text { cholesterol; CREA, creatinine; Na, sodium ions. }\end{array}$} \\
\hline
\end{tabular}

\subsection{Expression of p38 MAPK in ACLF}

LPS is known to engage p38 MAPK in inducing inflammatory response. We wondered whether this pathway participating in the pathogenesis of ACLF and tested in vivo expression of p38 MAPK in liver tissues from ACLF patients. Specimens displayed positive immunoreactions for p38 MAPK in patients with ACLF, and expressions of a-SMA were also found in specimens (Fig. 2A-H).

\subsection{LPS promotes inflammation in HSCs through p38 MAPK}

LPS is known to engage inflammatory response in THP-1 cells. We showed here that SB203580, a chemical inhibitor of p38 MAPK effectively suppressed LPS induced phosphorylation of p38 MAPK and NLRP3 inflammasomes activation in THP-1 cells (Fig. 3A, B, C). In the present study, we found that LPS could induce time-dependent activation of p38 MAPK, and SB203580 suppressed this phosphorylation (Fig. 3D, E) in LX2 cells. We also found that LPS induced a time-dependent activation of inflammatory 
response in LX2 cells, and the usage of SB203580 significantly attenuated LPS induced NLRP3 activation and production of IL-1 $\beta$ (Fig. 3F, G). In addition, we found a reversal of LPS induced accumulation of p62, which means autophagy inhibition, in SB203580 co-treated LX2 cells (Fig. 3F).

\subsection{LPS inhibits autophagy in HSCs through p38 MAPK}

To understand the mechanism of how signals control and release autophagic suppression of inflammation, we first tested whether LPS modulates autophagy in HSCs. We treated LX2 cells with LPS and found a and dose-dependent decrease in the level of autophagy marker LC3-II, the lipidated form of microtubule-associated protein 1A/1B-light chain 3 (LC3), and an increase in the level of autophagy adapter protein p62, respectively (Fig. 4A, B). Next, we evaluated the time course of autophagy in response to LPS in LX2 cells. LX2 cell were treated with $1 \mu \mathrm{g} / \mathrm{ml}$ of LPS, the LC3-II expression decreased, whereas p62 expression firstly increased and then decreased after 4 hours (Fig. 4C, D).

To further confirm that LPS inhibits autophagy in LX2 cell, we used rapamycin, an initiator of autophagy, and Bafilomycin A1 (Baf A1), which is known to block the fusion of autophagosomes with lysosomes, together with LPS. Our data showed that rapamycin inhibits LPS-induced changes in LC3-II and p62 (Fig. 4E). Exposure to Baf A1 significantly promoted LPS-induced accumulation of p62, and NLRP3 activation and production of IL-1 $\beta$ (Fig. 5G, H). Furthermore, SB203580 successfully alleviated LPSinduced inhibition of autophagy (Fig. 4F).

\section{5 p38 MAPK phosphorylates Atg13 and reduces Atg13- ULK1 interaction}

Inflammatory signal activates p38 MAPK, but its role has not been fully defined. Atg13 is a key regulator that functions upstream in the autophagic cascade and is regulated via phosphorylation, we tested the possibility that p38 MAPK may regulate autophagy by directly targeting Atg13. Here, we firstly found p38a MAPK may directly interact with Atg13 as we overexpressed MYC-Atg13 and FLAG-p38a MAPK in HEK293 cells, and immune-precipitated with an anti-MYC antibody and blotted the precipitate with an anti-FLAG antibody or performed the immunoprecipitation in reverse. This analysis showed that Atg13 and p38a MAPK directly associated with each other (Fig. 5A). Consistent with the possibility that p38a MAPK may regulate Atg13 activity, we found that co-expression with p38a MAPK causes Atg13 to migrate at a higher molecular weight position. This change in Atg13 migration could be reversed by pretreatment the lysates with SB203580 or $\lambda$ phosphatase (Fig. 5B, C). In LX2 cells, treatment of LPS led to phosphorylation and increased expression of Atg13 and this phenomenon was reversed by the usage of SB203580 (Fig. 5D).

Atg13 together with Atg101, FIP200 and ULK1 constitute the ULK1 complex, which plays an essential role in the initiation step of autophagy. Atg13 binds to ULK1 when receiving signals of nutrient status, and then recruits downstream autophagy related proteins. We tested whether p38 MAPK regulates the Atg13/ULK1 interaction. After treatment of the p38 MAPK activator anisomycin, the binding of Atg13 to ULK1 was decreased in HEK293 cells expressing MYC-tagged Atg13 and FLAG-tagged ULK1, suggesting 
that the inhibitory effects of p38 MAPK on autophagy were due to a decreased association of Atg13 with ULK1 that disrupted the initiation of autophagy. (Fig. 5E).

\section{Discussion}

It has been known for years that autophagy negatively control inflammasome activity, a decrease in autophagic activity correlates positively with inflammatory response[9]. ACLF is a life-threatening disease, characterized by over activation of hepatic inflammation[17]. Our data show that serum LPS level was significantly higher in ACLF due to increased gut permeability, meanwhile, serum LPS was found correlated with MELD score and associated with disease severity in patients with ACLF.

HSCs activation is the central step during liver fibrogenesis, our previous study revealed that HSCs activation participated in maintaining liver architecture during ALF[18]. Some recent studies have found that HSCs appear to replay inflammation signaling from the sinusoid to parenchyma: HSCs from both humans and rodents produce inflammatory cytokines that promote hepatocellular carcinoma and immune-mediated hepatitis[6]. However, few studies examined the roles of HSCs in hepatic inflammation during liver failure. Our recent work showed that during the pathogenesis of ALF, reactive oxygen species activate the NLRP3 inflammasome and promote inflammation in HSCs. We also revealed that LPS treatment induced reactive oxygen species (ROS) generation in HSCs via mitophagy inhibition[7]. In the present study, we found elevated expression of p38 MAPK in liver tissues from patients with ACLF. Stimulation of LPS inhibits autophagy via p38 MAPK, and this inhibition is necessary for LPS-induced inflammasome activation in LX2 cells. Our data suggested that usage of SB203580 successfully inhibits LPS induced autophagy inhibition and inflammasome activation.

We found in this present study a central mechanism by which proinflammatory signals exemplified by LPS relieves the tight inhibitory control exerted by autophagy on the inflammatory process. However, the mechanism through which this proinflammatory signal regulate autophagy in HSCs during ACLF remain elusive. We showed in the present study that in response to proinflammatory signal, p38 MAPK directly interacts with Atg13, phosphorylates Atg13 and reduces Atg13-ULK1 interaction, and then reduces autophagy[19]. Atg13 together with Atg101, FIP200 and ULK1 constitute the ULK1 complex, which plays an essential role in the initiation step of autophagy: receiving signals of nutrient status, recruiting downstream autophagy related proteins and governing autophagosome formation[20]. Because the Atg13-Ulk1 complex is the key upstream regulator of the autophagy pathway, our data suggests that proinflammatory signal engages autophagy at one of the earliest steps of entire process to allow more efficient removal of the inhibition during HSCs inflammation.

\section{Conclusion}

In conclusion, the present study revealed increased serum LPS level and overexpression of p38 MAPK in patients with ACLF. We also found LPS activates p38 MAPK, and then inhibits autophagy by disrupting 
the Atg13-ULK1 interaction in HSCs. Modulating of p38 MAPK or autophagy activity may be a novel strategy for treating patients with ACLF.

\section{Declarations}

Funding: This work was financially supported by the National Natural Science Foundation of China (81800548).

\section{Conflict of interest: None.}

Availability of data and materials: The datasets used and/or analyzed during the current study are available from the corresponding author on reasonable request.

Authors' contributions: Zhen Tian contributed to the study conception and design. Experiments were performed by Naijuan Yao, Fei Wang. Data analysis was performed by Zhen Tian and Yuchao Wu. The first draft of the manuscript was written by Zhen Tian and all authors commented on previous versions of the manuscript. All authors read and approved the final manuscript.

Ethics declarations: The study was approved by the Institutional Ethics Committee of The First Affiliated Hospital of Xi'an Jiaotong University and conforms to the ethical guidelines of the Declaration of HELSINKI.

\section{Acknowledgements}

This work was financially supported by the National Natural Science Foundation of China (81800548). The sponsors had no role in the study design and in the collection, analysis, and interpretation of data.

Zhen Tian wants to thank, in particular, for the love, support and patience from Jiajie Ma.

\section{References}

1. Bernal W, Jalan R, Quaglia A, Simpson K, Wendon J, Burroughs A. Acute-on-chronic liver failure. Lancet. 2015;386(10003):1576-87.

2. Zhao RH, Shi Y, Zhao H, Wu W, Sheng JF. Acute-on-chronic liver failure in chronic hepatitis B: an update. Expert Rev Gastroenterol Hepatol. 2018;12(4):341-50.

3. Blasco-Algora S, Masegosa-Ataz J, Gutiérrez-García ML, Alonso-López S, Fernández-Rodríguez CM. Acute-on-chronic liver failure: Pathogenesis, prognostic factors and management. World $\mathrm{J}$ Gastroenterol. 2015;21(42):12125-40.

4. Puche JE, Saiman Y, Friedman SL. Hepatic stellate cells and liver fibrosis. Compr Physiol. 2013;3(4):1473-92.

5. Zhai X, Wang W, Dou D, et al. A novel technique to prepare a single cell suspension of isolated quiescent human hepatic stellate cells. Sci Rep. 2019;9(1):12757. 
6. Fujita $\mathrm{T}$, Soontrapa $\mathrm{K}$, Ito $\mathrm{Y}$, et al. Hepatic stellate cells relay inflammation signaling from sinusoids to parenchyma in mouse models of immune-mediated hepatitis. Hepatology. 2016;63(4):1325-39.

7. Tian $Z$, Chen $Y$, Yao N, et al. Role of mitophagy regulation by ROS in hepatic stellate cells during acute liver failure. Am J Physiol Gastrointest Liver Physiol. 2018;315(3):G374-4G384.

8. Levine B, Kroemer G. Autophagy in the pathogenesis of disease. Cell. 2008;132(1):27-42.

9. Matsuzawa-Ishimoto Y, Hwang S, Cadwell K. Autophagy and Inflammation. Annu Rev Immunol. 2018;36:73-101.

10. Ilyas G, Cingolani F, Zhao E, Tanaka K, Czaja MJ. Decreased Macrophage Autophagy Promotes Liver Injury and Inflammation from Alcohol. Alcohol Clin Exp Res. 2019;43(7):1403-13.

11. Xiong X, Ren Y, Cui Y, Li R, Wang C, Zhang Y. Obeticholic acid protects mice against lipopolysaccharide-induced liver injury and inflammation. Biomed Pharmacother. 2017;96:1292-8.

12. Gaestel M. MAPK-Activated Protein Kinases (MKs): Novel Insights and Challenges. Front Cell Dev Biol. 2015;3:88.

13. Yeung YT, Aziz F, Guerrero-Castilla A, Arguelles S. Signaling Pathways in Inflammation and Antiinflammatory Therapies. Curr Pharm Des. 2018;24(14):1449-84.

14. Yong HY, Koh MS, Moon A. The p38 MAPK inhibitors for the treatment of inflammatory diseases and cancer. Expert Opin Investig Drugs. 2009;18(12):1893-905.

15. Kongdang P, Jaitham R, Thonghoi S, Kuensaen C, Pradit W, Ongchai S. Ethanolic extract of Kaempferia parviflora interrupts the mechanisms-associated rheumatoid arthritis in SW982 culture model via p38/STAT1 and STAT3 pathways. Phytomedicine. 2019;59:152755.

16. Feng YJ, Li YY. The role of p38 mitogen-activated protein kinase in the pathogenesis of inflammatory bowel disease. J Dig Dis. 2011;12(5):327-32.

17. Triantafyllou E, Woollard KJ, MJW M, Antoniades CG, Possamai LA. The Role of Monocytes and Macrophages in Acute and Acute-on-Chronic Liver Failure. Front Immunol. 2018;9:2948.

18. He Y, Jin L, Wang J, Yan Z, Chen T, Zhao Y. Mechanisms of fibrosis in acute liver failure. Liver Int. 2015;35(7):1877-85.

19. Kaizuka T, Mizushima N. Atg13 Is Essential for Autophagy and Cardiac Development in Mice. Mol Cell Biol. 2016;36(4):585-95.

20. Park JM, Jung $\mathrm{CH}$, Seo M, et al. The ULK1 complex mediates MTORC1 signaling to the autophagy initiation machinery via binding and phosphorylating ATG14. Autophagy. 2016;12(3):547-64.

\section{Figures}


A
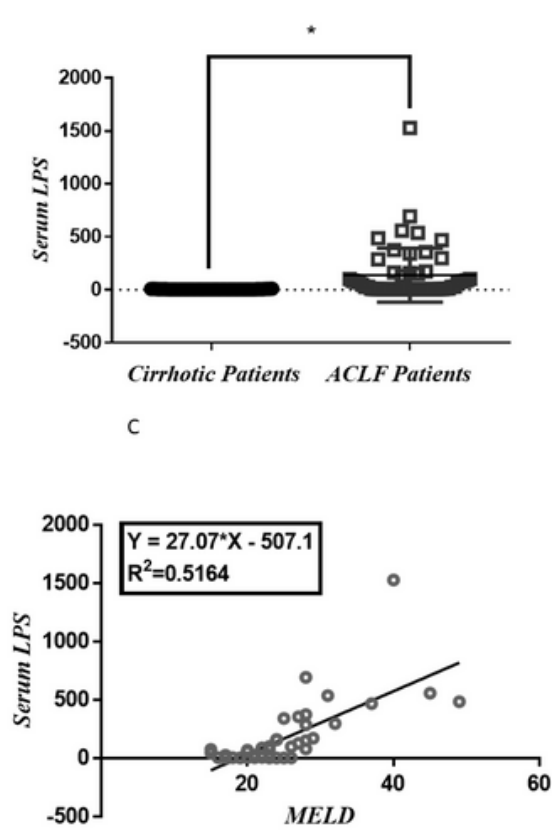

B

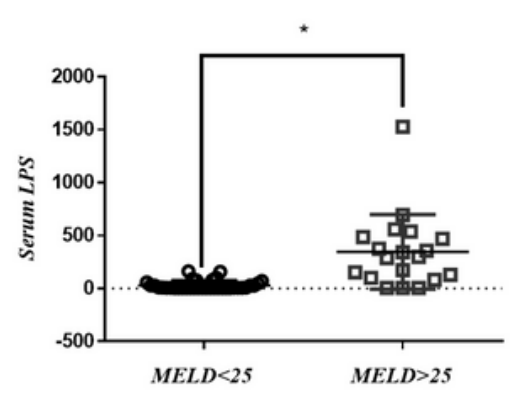

\section{Figure 1}

Increased serum LPS level is associated with poor prognosis of ACLF. Plasma from acute-on-chronic liver failure (ACLF) patients at admission and from healthy controls were tested for LPS. (A) LPS level in ACLF patients was significantly increased compared to healthy controls. (B) ACLF patients with MELD score $>25$ displayed higher LPS level compared to patients with MELD score $\leq 25$. (C) Positive correlation between serum LPS level and MELD score. ${ }^{*} \mathrm{P}<0.01$. 


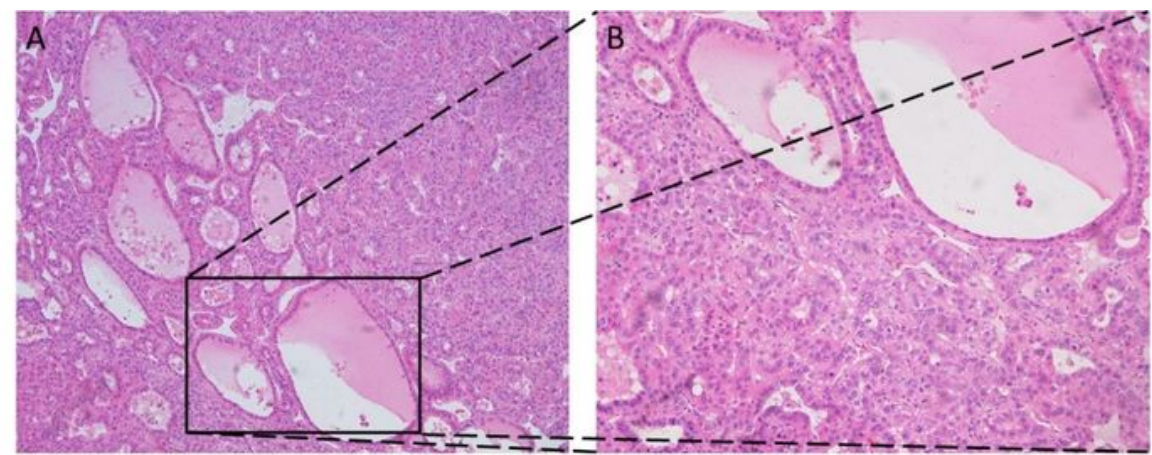

ACLF HE 100 $\times$

ACLF HE 200x

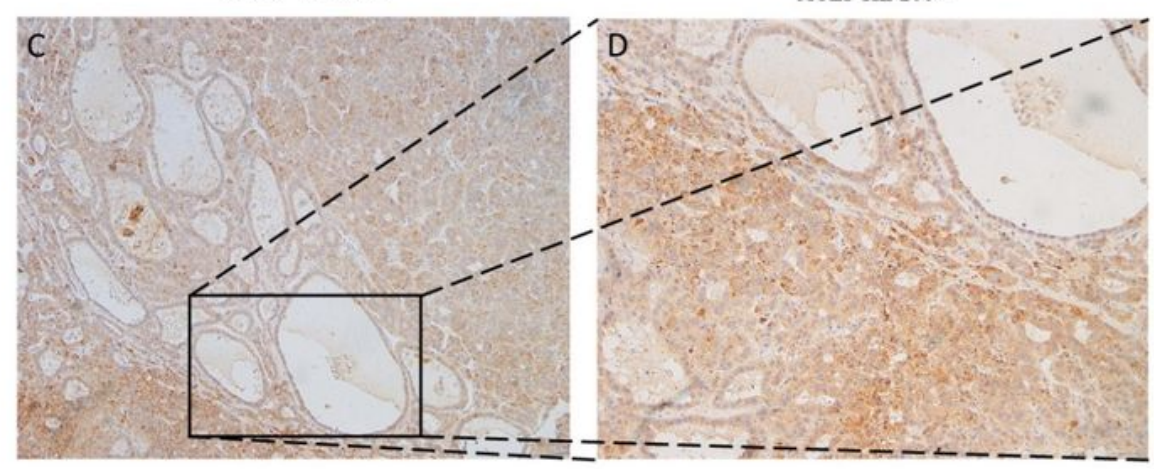

ACLF $\alpha$-SMA $100 \times$

ACLF $\alpha$-SMA $200 \times$

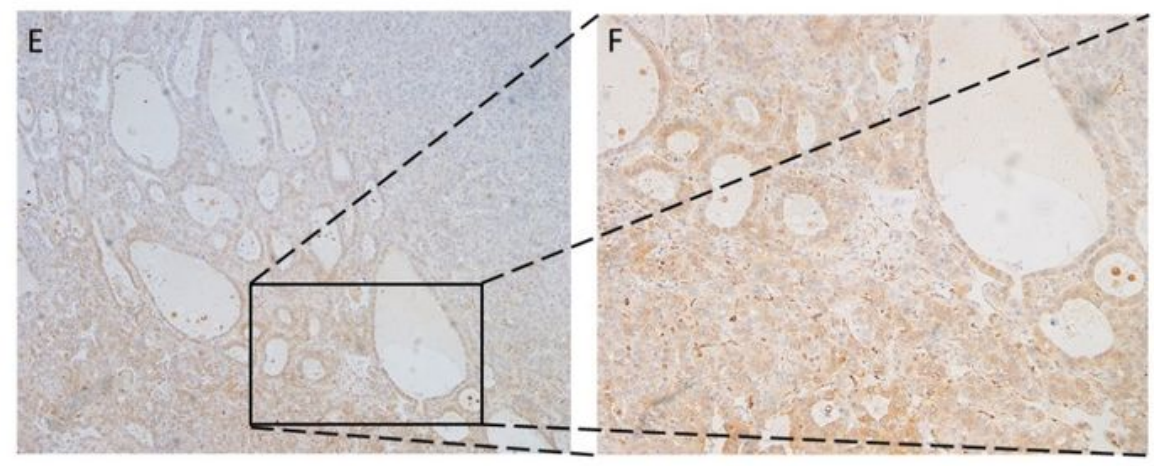

ACLF p38 MAPK 100x

ACLF p38 MAPK 200×

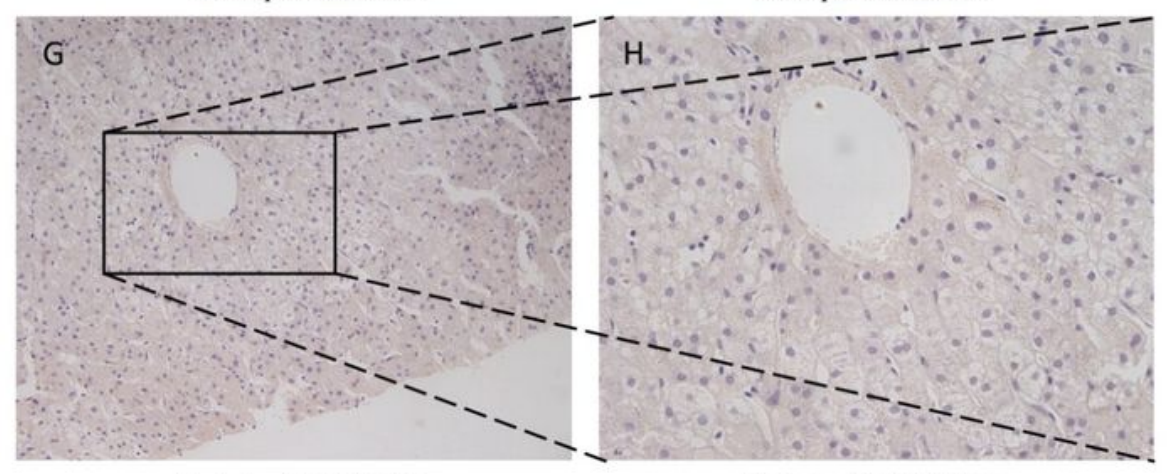

Cirrhosis p38 MAPK 100x

Cirrhosis p38 MAPK $200 \times$

\section{Figure 2}

Immunohistochemical staining were conducted in liver tissues from patients with ACLF and cirrhosis. The tissues from ACLF patients were firstly stained with hematoxylin-eosin (HE) (A, B), then the expression of a-SMA (C, D) and p38 MAPK (E, F). The tissues from cirrhotic patients were stained with p38 MAPK. Original magnification $100 \times(A, B, C$ and $G)$ or $200 \times(D, E, F$ and $H)$. 
A

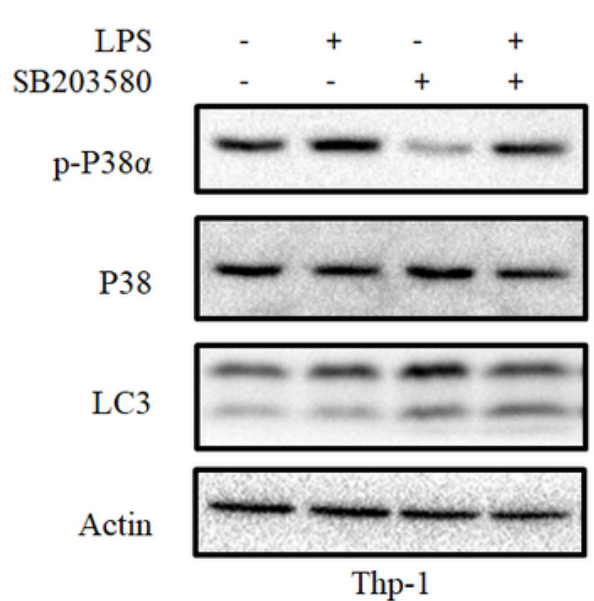

D

LPS

$$
\text { p-P38 } \alpha
$$

$\begin{array}{llllllll}0 & 7 \mathrm{~m} & 15 \mathrm{~m} & 30 \mathrm{~m} & 1 \mathrm{~h} & 2 \mathrm{~h} & 4 \mathrm{~h} & 8 \mathrm{~h}\end{array}$

\section{P38}
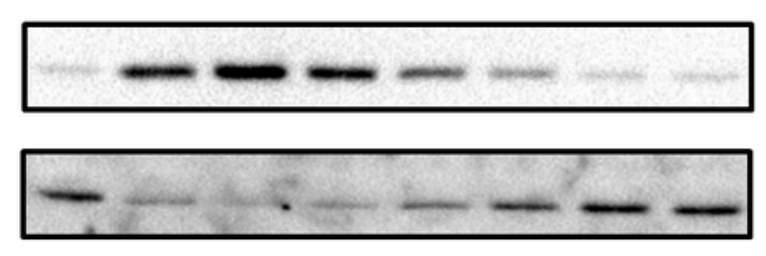

Actin

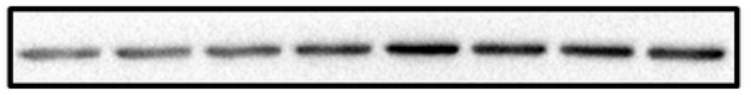

F

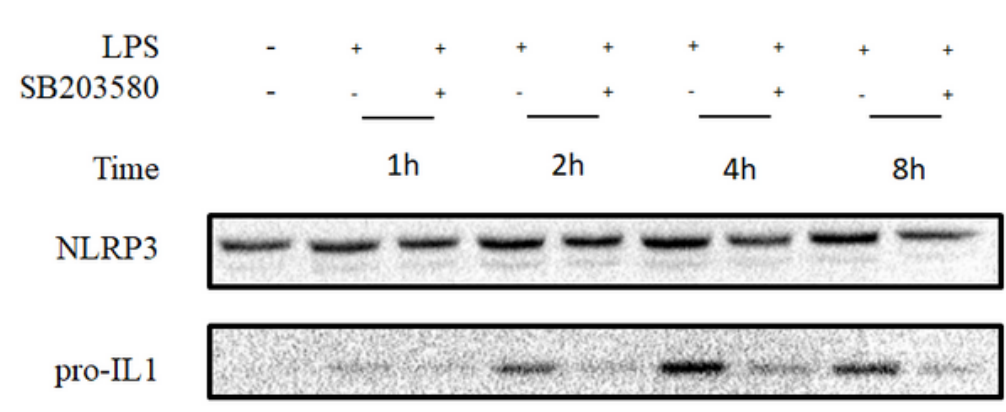

P62

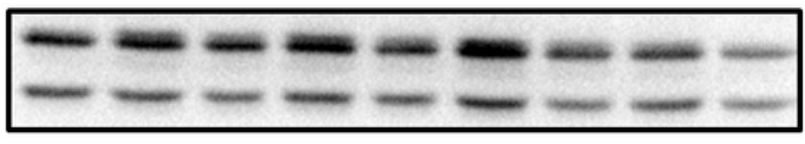

Actin

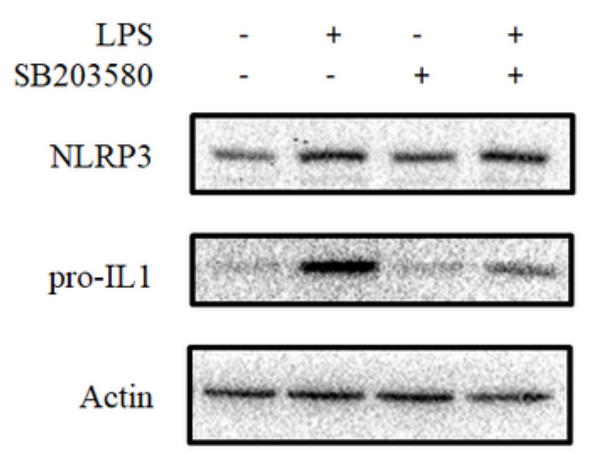

Thp-1

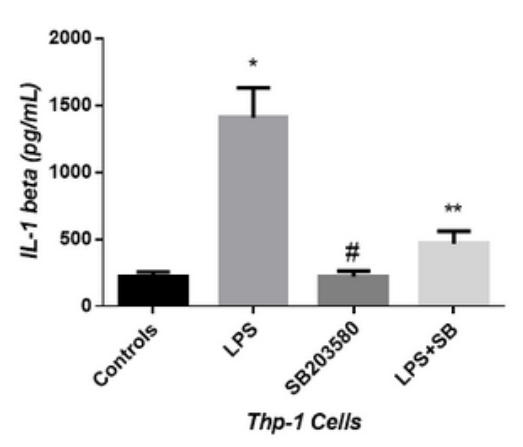

E

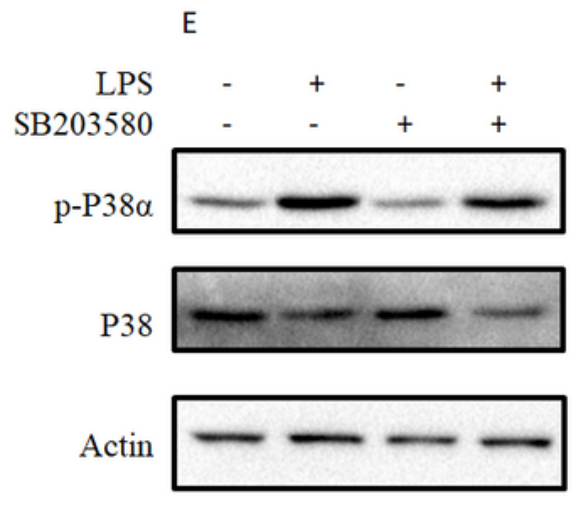

G

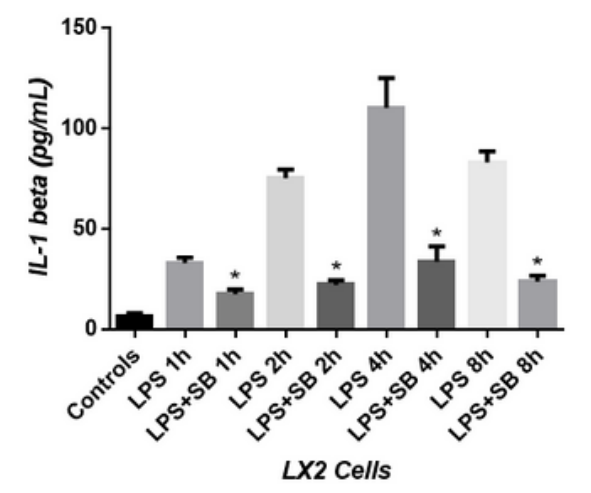

\section{Figure 3}

LPS promotes inflammation via p38 MAPK in HSCs. (A-C) Thp-1 cells in the logarithmic growth phase were treated with $1 \mu \mathrm{g} / \mathrm{ml}$ LPS for $15 \mathrm{~min}$ for the detection of p38 MAPK or for $4 \mathrm{~h}$ for the detection of inflammation; (D) LX-2 cells in the logarithmic growth phase were treated with $1 \mu \mathrm{g} / \mathrm{ml}$ LPS in a timedependent manner (0-8h); (E-G) LX-2 cells in the logarithmic growth phase were treated with or without 20 $\mu \mathrm{M}$ SB203580 for $30 \mathrm{~min}$, then cells were treated with $1 \mu \mathrm{g} / \mathrm{ml}$ LPS for $15 \mathrm{~min}$ for the detection of p38 
MAPK or in a time-dependent manner (0-8h) for the detection of inflammation. Then, inflammation was evaluated by immunoblotting with specific antibodies, as indicated. The NLRP3 and pro-IL1 levels were quantified by densitometry. Secretion levels of IL-1 $\beta$ were detected by ELISA.

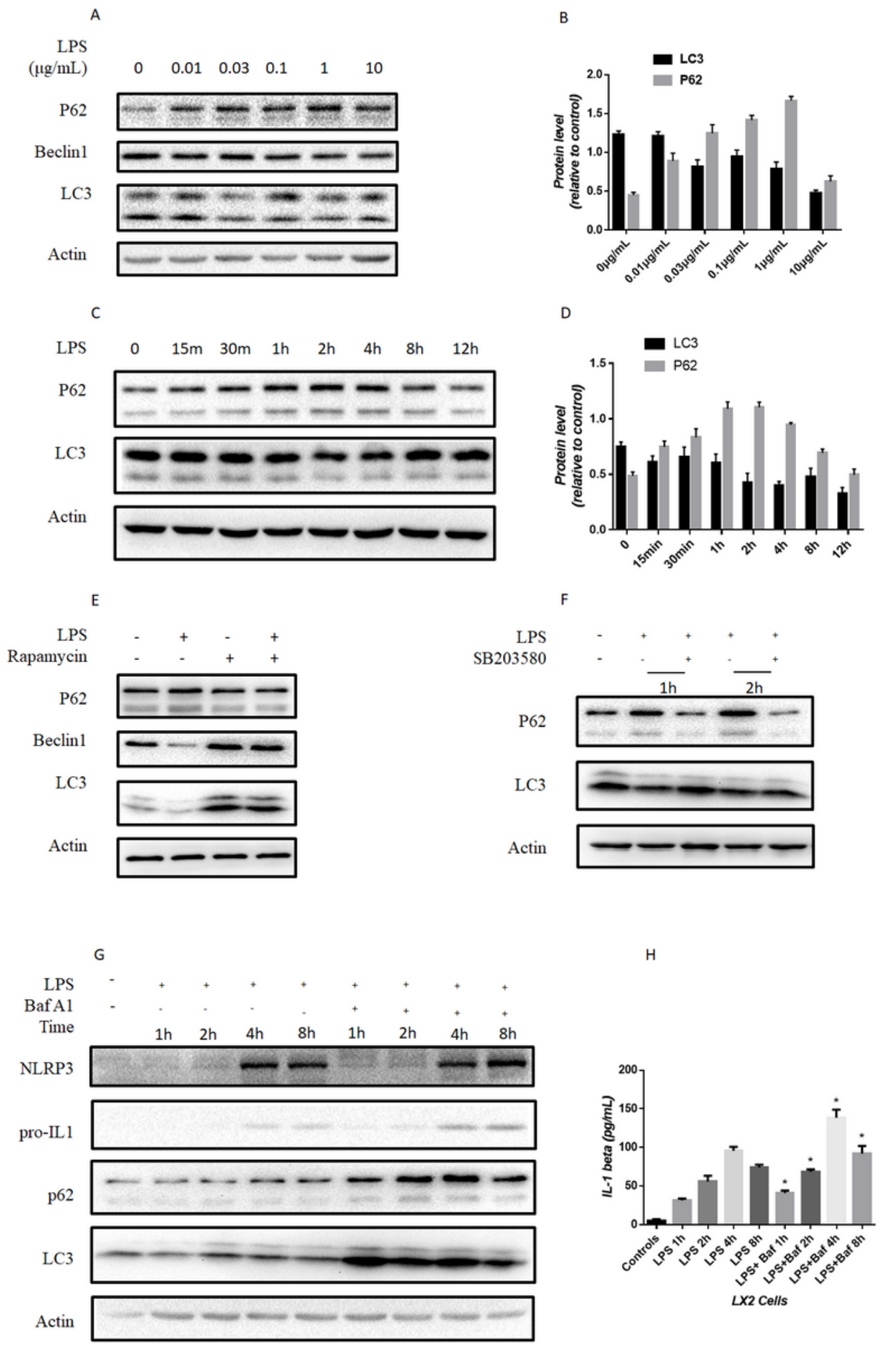

Figure 4

LPS inhibits autophagy via p38 MAPK in HSCs. (A, B) LX-2 cells in the logarithmic growth phase were treated with different concentrations of LPS $(0-10 \mu \mathrm{g} / \mathrm{ml})$ for $4 \mathrm{~h}$; (C, D) LX-2 cells in the logarithmic 
growth phase were treated with $1 \mu \mathrm{g} / \mathrm{ml}$ LPS in a time-dependent manner (0-8h); $(\mathrm{E}, \mathrm{F}) \mathrm{LX}-2$ cells in the logarithmic growth phase were treated with $1 \mu \mathrm{g} / \mathrm{ml}$ LPS together with or without 50nM Rapamycin or $20 \mu \mathrm{M}$ SB203580 for 4h for the detection of autophagy; $(G, H)$ LX-2 cells in the logarithmic growth phase were treated with $1 \mu \mathrm{g} / \mathrm{ml}$ LPS together with or without 200nM Bafilomycin $A$ in a time-dependent manner $(0-8 \mathrm{~h})$ for the detection of autophagy. Then, autophagy and inflammation were evaluated by immunoblotting with specific antibodies, as indicated. The P62, LC3, NLRP3 and pro-IL1 levels were quantified by densitometry and normalized to the actin level. Secretion levels of IL-1 $\beta$ were detected by ELISA. 

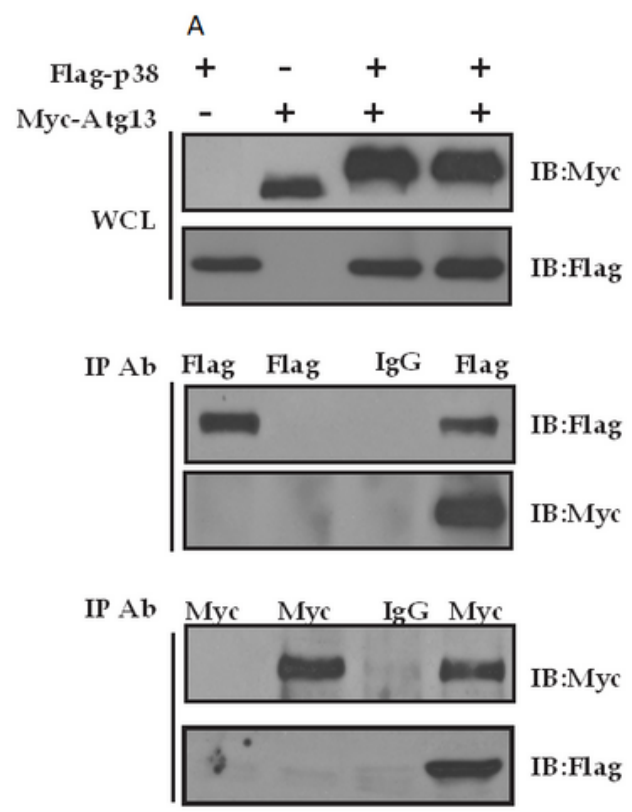

C

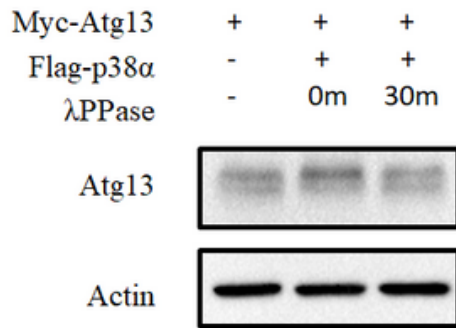

$E$

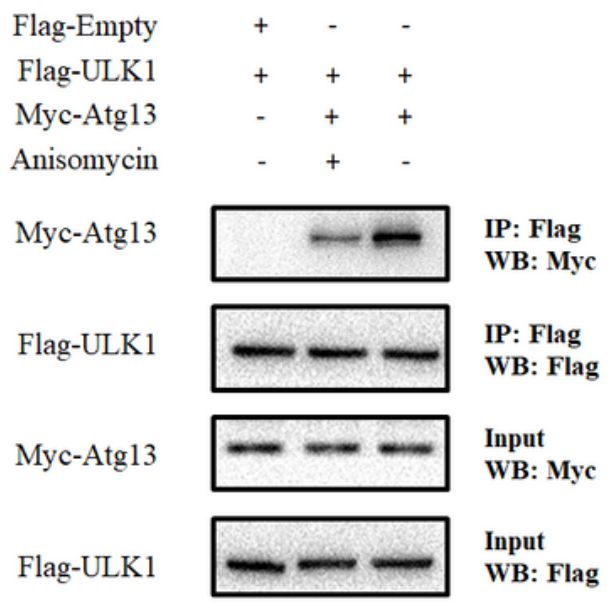

B

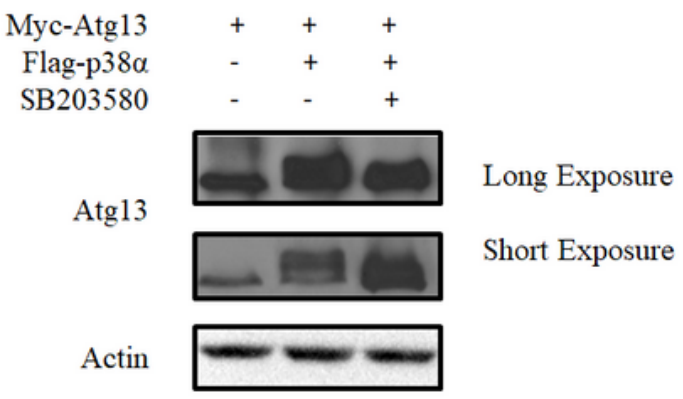

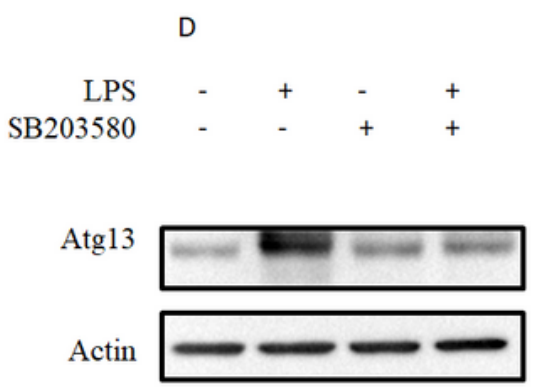

\section{Figure 5}

p38 MAPK interacts with and phosphorylates Atg13 (A) Myc-tagged Atg13 and Flag-tagged p38 were expressed in HEK-293 cells. $36 \mathrm{~h}$ following transfection, cell lysate was immunoprecipitated with anti-Myc and anti-Flag antibodies; (B, C) Atg13 and p38 were expressed in HEK-293 cells. 36h following transfection, transfected HEK-293 cells were subjected to $20 \mu \mathrm{M}$ SB203580 or $\lambda$ phosphatase treatment for $30 \mathrm{~min}$, then the expression of Atg13 was detected. (D) LX-2 cells in the logarithmic growth phase were 
treated with or without $20 \mu \mathrm{M}$ SB203580 for $30 \mathrm{~min}$ before the usage of $1 \mu \mathrm{g} / \mathrm{ml}$ LPS for $1 \mathrm{~h}$, the expression of Atg13 was then detected. (E) Myc-tagged Atg13 and Flag-tagged p38 were expressed in HEK-293 cells with or without anisomycin. 36h following transfection, cell lysate was immunoprecipitated with anti-Myc and anti-Flag antibodies. Then, expression of p38 MAPK, Atg13 and ULK1 were evaluated by immunoblotting with specific antibodies, as indicated. 